PROCEEDINGS OF THE

AMERICAN MATHEMATICAL SOCIETY

Volume 140, Number 5, May 2012, Pages 1803-1813

S 0002-9939(2011)11262-0

Article electronically published on September 6, 2011

\title{
CONCENTRATION FOR NONCOMMUTATIVE POLYNOMIALS IN RANDOM MATRICES
}

\author{
MARK W. MECKES AND STANISŁAW J. SZAREK
}

(Communicated by Marius Junge)

\begin{abstract}
We present a concentration inequality for linear functionals of noncommutative polynomials in random matrices. Our hypotheses cover most standard ensembles, including Gaussian matrices, matrices with independent uniformly bounded entries and unitary or orthogonal matrices.
\end{abstract}

\section{INTRODUCTION}

The starting point of this paper was an inquiry of W. Bryc concerning almost sure convergence for certain non-Gaussian matrix models in free probability. Almost sure convergence questions often reduce to concentration inequalities, which may be interesting in their own right, and our purpose is to present one such inequality.

Our approach is as follows. We start by defining the convex concentration property (CCP) of normed-space-valued random variables. When specialized to random matrices, the class CCP contains most standard ensembles, in particular the (appropriately normalized) Wigner-type matrices with independent bounded entries that were the object of Bryc's inquiry. Then we state and prove a concentration inequality for noncommutative polynomials in independent random matrices verifying the CCP.

This approach was inspired by the results of M. Talagrand [28, 29, 30] on concentration of measure in product spaces. These tools were first adapted to the random matrix context by Guionnet and Zeitouni in [11] and by Krivelevich and Vu in [17, with subsequent applications in [2, 21. However, various features of the present setup (noncommutativity, nonselfadjointness, the absence of the Lipschitz property in polynomials of degree greater than 1) do not fit into the standard framework, and, consequently, a few additional tricks will be required.

\section{Convex COnCEntration PRoperty}

We say that a random vector $X$ in a normed space $V$ satisfies the (subgaussian) convex concentration property $(\mathrm{CCP})$ or is in the class $\mathrm{CCP}$ if

$$
\mathbb{P}[|f(X)-\mathbb{M} f(X)| \geq t] \leq K e^{-\kappa t^{2}}
$$

for every $t>0$ and every convex 1-Lipschitz function $f: V \rightarrow \mathbb{R}$, where $K, \kappa>0$ are constants (parameters) independent of $f$ and $t$, and $\mathbb{M}$ denotes a median of a

Received by the editors January 17, 2011.

2010 Mathematics Subject Classification. Primary 60E15, 60B20.

(C)2011 American Mathematical Society

Reverts to public domain 28 years from publication 
random variable. Even though this was not explicitly defined, this property already made an appearance in 28. The class CCP enjoys various stability properties, for example if $X, Y$ satisfy the CCP, so does their concatenation $(X, Y)$ (as follows from the proof of [19, Proposition 1.11]). Clearly, various generalizations of the concept are possible. For example, one may consider tail behaviors other than subgaussian or allow other classes of test functions $f$; see, e.g., 1 .

While the subgaussian tail condition in (1) may appear stringent, it is verified by many natural classes of multivariate distributions. For example, if $V=\mathbb{R}^{N}$ and the components $X_{i}$ are independent normal random variables with uniformly bounded variances or if the random variables $\left(X_{i}-\mathbb{E} X_{i}\right)$ are uniformly bounded ( $\mathbb{E}$ stands for the expected value of a random variable), then $X$ satisfies the CCP. Examples with dependent components include $X$ uniform on $\sqrt{N} S^{N-1}$ or with a density proportional to $e^{-u(x)}$, where the Hessian of $u$ verifies $D^{2} u \geq c I, c>0$. See [19] for multiple proofs of all these statements and much more information, and [1] for a discussion of various fine points concerning the class CCP. Here we will just mention that the validity of the first example is a consequence of Borell-SudakovTsirelson Gaussian isoperimetric inequality, the second one is the primary instance of Talagrand's approach to concentration on product spaces, the third one follows from Paul Lévy's spherical isoperimetric inequality, and the last is a consequence of the theory of logarithmic Sobolev inequalities. We emphasize that the common and crucial feature of all these examples, and of others that will follow, is dimension independence: while the parameters $K, \kappa$ in (10) may depend on the characteristics of the family in question (for instance, on the bound on variances implicit in the first example above or on the value of $c$ in the last example), they do not depend on the dimension of the underlying vector space.

As is well-known and easy to check, a concentration inequality of type (1) implies that the mean and median of $f(X)$ differ by at most a constant (depending only on the parameters $K, \kappa$; see, e.g., [19, Section 1.3] or [22, Proposition V.4]). It follows that concentration about the median is equivalent to concentration about the mean up to modification of the constants in (11). At different points in the results and proofs below it will be convenient to work with either the mean or the median.

\section{Matrix ensembles: The Main Result}

We denote by $M_{n}$ the space of $n \times n$ complex matrices, by $M_{n}^{s a}$ its (real vector) subspace of Hermitian matrices, and by $\|A\|_{p}:=\left(\operatorname{tr}\left(A^{*} A\right)^{p / 2}\right)^{1 / p}$ the Schatten $p$ norm of a matrix $A$. The limiting case $p=\infty$ corresponds to the operator (or spectral) norm, while $p=2$ leads to the Hilbert-Schmidt (or Frobenius) norm. We also denote by $\|\cdot\|_{p}$ the $L_{p}$-norm of a (real or complex) random variable or the $\ell_{p}$-norm of a vector in $\mathbb{R}^{N}$ or $\mathbb{C}^{N}$. Below and in what follows $C, C_{1}, C^{\prime}, c$, etc., stand for positive numerical constants whose value may change from line to line. Similarly (for example) $c_{d, m}$ will denote a positive constant which may depend on the parameters $d$ and $m$, but not on the underlying dimension. Such constants will in general depend implicitly on the parameters $K, \kappa$ in (11) and, if applicable, on other constants appearing in the hypotheses of a particular statement. This dependence will be straightforward to make explicit, but for the sake of simplicity we have mostly not chosen to do so here. 
Theorem 1. Let $X_{1}, \ldots, X_{m} \in M_{n}$ be independent centered random matrices which satisfy the convex concentration property (with respect to the Hilbert-Schmidt norm on $M_{n}$ ) and let $d \geq 1$ be an integer. Let $P$ be a noncommutative *-polynomial in $m$ variables of degree at most $d$, normalized so that its coefficients have modulus at most 1 . Define the complex random variable

$$
Z_{P}=\operatorname{tr} P\left(\frac{X_{1}}{\sqrt{n}}, \ldots, \frac{X_{m}}{\sqrt{n}}\right) .
$$

Then, for $t>0$,

$$
\mathbb{P}\left[\left|Z_{P}-\mathbb{E} Z_{P}\right| \geq t\right] \leq C_{m, d} \exp \left[-c_{m, d} \min \left\{t^{2}, n t^{2 / d}\right\}\right] .
$$

The conclusion holds also for noncentered random matrices if - when $d \geq 2-$ we assume that $\left\|\mathbb{E} X_{j}\right\|_{2(d-1)} \leq C n^{d / 2(d-1)}$ for all $j$.

It is a standard observation that, by integration by parts on the one hand and the Bienaymé-Chebyshev-Markov inequality on the other hand, a tail bound as in Theorem 1 is equivalent to a bound on the growth of $L_{p}$-norms.

Corollary 2. Let $Z_{P}$ be as in Theorem 1. Then for $q \geq 1$,

$$
\left\|Z_{P}-\mathbb{E} Z_{P}\right\|_{q} \leq C_{m, d}^{\prime} \max \left\{\sqrt{q},\left(\frac{q}{n}\right)^{d / 2}\right\} .
$$

Remarks. 1. The hypotheses of Theorem 1 cover Wigner-type matrices with independent Gaussian or independent bounded entries, but not arbitrary independent subgaussian entries (see 1] and its references; note that CCP clearly implies that the entries are subgaussian). However, independent entries satisfying a logarithmic Sobolev inequality, or more generally a quadratic transportation cost inequality, are covered (see [19, Chapters 5-6]). Moreover, the hypotheses also cover many cases with dependent matrix entries. The most notable are the following:

(a) $X_{j}$ drawn from an orthogonal or unitary ensemble, that is, with a density w.r.t. Lebesgue measure on $M_{n}^{s a}$ proportional to $e^{-\operatorname{tr} u(X)}$, in the case that $u: \mathbb{R} \rightarrow \mathbb{R}$ satisfies $u^{\prime \prime} \geq c>0$. (This again follows from the theory of logarithmic Sobolev inequalities.) Ensembles of this form are widely studied in the literature (see, e.g., [8]). In the context of nuclear physics this is a more natural class than that of Wigner matrices.

(b) $X_{j}$ such that $n^{-1 / 2} X_{j}$ is uniformly distributed in the special orthogonal or unitary group (see [22, Section 6] or [19, Section 2.1]).

(c) $X_{j}$ uniformly distributed on the (Hilbert-Schmidt) sphere of $M_{n}^{s a}$ of radius $\sqrt{\frac{n(n+1)}{2}}$ or $n$ in the real case or complex case, respectively, or uniformly distributed on the sphere of radius $n$. (In fact, any $O(n)$ radii would do, but the exact values we cite here appear in a natural way.)

2. A perhaps more natural way to state the bound on $\mathbb{E} X_{j}$ in the noncentered case (if each $X_{j}$ is Hermitian) is

$$
\operatorname{tr}\left(\mathbb{E} \frac{X_{j}}{\sqrt{n}}\right)^{2(d-1)} \leq C n
$$

A slightly stronger simple hypothesis is $\left\|\mathbb{E} X_{j}\right\|_{\infty} \leq C \sqrt{n}$. 
3. It is not strictly necessary that the $X_{j}$ be independent, only that the joint distribution of $\left(X_{1}, \ldots, X_{m}\right) \in \bigoplus_{j=1}^{m} M_{n}$ satisfy the convex concentration property, with constants that may depend on $m$.

4. When $d>2$, it suffices for the proof to assume that $X_{j}$ satisfies the convex concentration property with respect to the Schatten norm $\|\cdot\|_{d}$ on $M_{n}$, but it is not clear whether this is a useful observation.

\section{The BACKGRound AND The CONSEQUENCES}

Here is a consequence of Theorem 1 in the spirit of the original inquiry of Bryc. For simplicity, we state it in the real case only.

Corollary 3. Let $X_{1}, \ldots, X_{m}, P$ and $Z_{P}$ be as in Theorem 1, and assume further that, for each $j, X_{j}$ is real symmetric and its upper-diagonal entries are independent and of unit variance. Then, almost surely,

$$
n^{-1} Z_{P} \rightarrow \tau\left(P\left(a_{1}, a_{2}, \ldots, a_{m}\right)\right)
$$

where $a_{1}, a_{2}, \ldots, a_{m}$ are free semicircular elements in a noncommutative probability $\operatorname{space}(\mathcal{A}, \tau)$.

The connection between random matrices and free probability was established in the seminal paper [33], where the weaker convergence $n^{-1} \mathbb{E} Z_{P} \rightarrow \tau\left(P\left(a_{1}, a_{2}, \ldots, a_{m}\right)\right)$ was shown in the Gaussian case (we refer to [34, 10] for more background on free probability). This was generalized to (in particular) other Wigner-like ensembles in [9] and strengthened in various ways in [12, 27, 4, 24, 23, 20.

The fact that the weaker convergence (of expected values) in combination with concentration (which was known for Gaussian and some other classical ensembles) implies almost sure convergence was essentially folklore (see [13, 14, 5]): the deviation of $n^{-1} Z_{P}$ from its expected value has a tail that decays (at least) exponentially in $n$; hence the Borel-Cantelli lemma applies. Note that rescaling by $n^{-1}$ is appropriate since the noncommutative probability context calls for the normalized trace $n^{-1}$ tr.

The same argument applies to any other ensemble which verifies the CCP and for which the limit object — in the (weak) noncommutative probability sense exists. On the other hand, results along the lines of Corollary 3 can also be proved without Theorem [1 and in particular under weaker assumptions than exponential concentration. Theorem 2 of 25 proves what amounts to the conclusion of Corollary 3 for Wigner matrices with i.i.d. entries with bounded fourth moments; see 25] for references to earlier results proved under stronger assumptions. In addition, concentration inequalities for some noncommutative functionals of random matrices — but not polynomials — appeared already in 11] (Theorem 1.9; the entries are required to satisfy logarithmic Sobolev inequality).

Finally, let us point out that there is a fairly extensive literature on the tail behavior of "higher order chaoses" (i.e., polynomials) in classical probability, i.e., without focus on the issues related to the matrix structure or noncommutativity, for example [7, 18, 1]. There are also applications of concentration of polynomials to combinatorics [15, 16, 35. 


\section{The Proof: A special Case}

Theorem 1 will be deduced from the special case of a power of a single Hermitian random matrix.

Proposition 4. Let $X \in M_{n}^{s a}$ be a random Hermitian matrix which satisfies the convex concentration property (with respect to the Hilbert-Schmidt norm on $M_{n}^{s a}$ ), let $d \geq 1$ be an integer, and suppose - when $d \geq 2$ - that $\operatorname{tr}\left(\mathbb{E} \frac{X}{\sqrt{n}}\right)^{2(d-1)} \leq C n$. Then for $t>0$,

$$
\mathbb{P}\left[\left|\operatorname{tr}\left(\frac{X}{\sqrt{n}}\right)^{d}-\mathbb{M} \operatorname{tr}\left(\frac{X}{\sqrt{n}}\right)^{d}\right| \geq t\right] \leq C \exp \left[-\min \left\{c^{d} t^{2}, c n t^{2 / d}\right\}\right] .
$$

The essential idea in the proof of this concentration inequality is of course to apply the CCP to the functional $A \mapsto \operatorname{tr} A^{d}$, but there are two obvious difficulties with this approach. One is that this functional is not convex if $d$ is odd and $d \geq 3$, and the convexity is not entirely trivial when $d$ is even. This technicality is readily dealt with by using a classical convexity lemma and (in the odd case) a simple decomposition trick. The second, more fundamental problem is that when $d \geq 2$ this functional is not Lipschitz (in fact, not even uniformly continuous). However, it is locally Lipschitz in a way which is readily quantified so that a variation of standard truncation arguments can be applied. Extra care is needed here to show that the truncation procedure can be made to preserve the convexity of the functional and its Lipschitz constant and also to control the effect of the truncation on the median. The following folklore result will be helpful.

Lemma 5. Let $V$ be finite-dimensional normed space, $K \subseteq V$ an open convex set, and $F: K \rightarrow \mathbb{R}$ a convex Lipschitz function. Then there exists a function $\widetilde{F}: V \rightarrow \mathbb{R}$ such that:

- $\widetilde{F}$ is convex and $\left.\widetilde{F}\right|_{K}=F$ (i.e., $\widetilde{F}$ is a convex extension of $F$ );

- $\widetilde{F}$ is pointwise minimal among all convex extensions of $F$; and

- $\widetilde{F}$ is Lipschitz, and its Lipschitz constant is the same as that of $F$.

Proof. For $y \in K$, recall that (cf. [26, Section 23])

$$
\partial F(y)=\left\{\phi \in V^{*} \mid F(x) \geq F(y)+\phi(x-y)\right\}
$$

is the subdifferential of $F$ at $x$ (nonempty because $F$ is convex) so that

$$
F(x)=\sup \{F(y)+\phi(x-y) \mid \phi \in \partial F(y), y \in K\}
$$

for every $x \in K$. Moreover, the Lipschitz constant of $F$ (on $K$ ) is

$$
\sup \{\|\phi\| \mid \phi \in \partial F(y), y \in K\}
$$

(cf. [26, Corollary 13.3.3]). This implies that the supremum in (2) is finite also for $x \notin K$ and thus defines an extension $\widetilde{F}: V \rightarrow \mathbb{R}$. The assertions of the lemma follow easily from this definition.

Proof of Proposition 4. The case $d=1$ is an immediate consequence of the CCP (1), so we will assume from now on that $d \geq 2$.

Let $F: M_{n}^{s a} \rightarrow \mathbb{R}$ be given by

$$
F(A)=\operatorname{tr} A^{d}=\sum_{i=1}^{n} \lambda_{i}(A)^{d}
$$


where $\lambda_{i}(A)$ are the eigenvalues of $A$ in, say, nonincreasing order. A classical lemma of matrix analysis (see e.g. 3, Lemma 4.4.12]) states that a functional $A \mapsto \operatorname{tr} \phi(A)$ is convex whenever $\phi: \mathbb{R} \rightarrow \mathbb{R}$ is convex; hence in particular our $F$ is convex when $d$ is even. If $d \geq 3$ is odd, then we can write $F(A)=F^{+}(A)-F^{-}(A)$, where

$$
F^{ \pm}(A)=\sum_{i=1}^{n} \lambda_{i}(A)_{ \pm}^{d}
$$

Here $x_{+}=\max \{0, x\}$ and $x_{-}=\max \{0,-x\}$. Since both the functions $x \mapsto x_{ \pm}^{d}$ are convex, $F^{ \pm}: M_{n}^{s a} \rightarrow \mathbb{R}$ are both convex. In the rest of this proof, for clarity of exposition, we will proceed as if $d$ is even. The odd case is handled in the same way by considering $F^{+}$and $F^{-}$separately, then deducing the concentration of

$$
F(X)-\mathbb{E} F(X)=\left(F^{+}(X)-\mathbb{E} F^{+}(X)\right)-\left(F^{-}(X)-\mathbb{E} F^{-}(X)\right)
$$

from the concentration of each summand and the triangle inequality.

Let $f: \mathbb{R}^{n} \rightarrow \mathbb{R}$ be given by $f(x)=\sum_{i=1}^{n} x_{i}^{d}$. Another classical lemma of matrix analysis (see e.g. 3. Lemma 2.1.19 and Remark 2.1.20]) states that the map $A \mapsto\left(\lambda_{1}(A), \ldots, \lambda_{n}(A)\right)$ is 1-Lipschitz from $M_{n}^{s a}$ with the Hilbert-Schmidt norm to $\mathbb{R}^{n}$ with the standard Euclidean norm. The local Lipschitz behavior of $F$ can therefore be controlled via the local Lipschitz behavior of $f$, for which we compute

$$
|\nabla f(x)|=\sqrt{d^{2} \sum_{i=1}^{n} x_{i}^{2(d-1)}}=d\|x\|_{2(d-1)}^{d-1} .
$$

We now describe our truncation procedure. For each $a>0$, we set

$$
K_{a}=\left\{A \in M_{n}^{s a} \mid\|A\|_{2(d-1)}<a\right\} ;
$$

then $\left.F\right|_{K_{a}}$ is $\left(d a^{d-1}\right)$-Lipschitz. At this point we appeal to Lemma 5 to obtain convex $\left(d a^{d-1}\right)$-Lipschitz extensions $F_{a}: M_{n}^{s a} \rightarrow \mathbb{R}$ to which the CCP applies. Moreover, since $\left\{K_{a}\right\}$ is a nested family of open convex sets whose union is $M_{n}^{s a}$, the minimality property from Lemma 5 implies that, for each $A \in M_{n}^{s a}, F_{a}(A)$ increases to $F(A)$ as $a \rightarrow \infty$.

The other necessary ingredient for the truncation-type argument is an upper bound on the probability of the event that $X \notin K_{a}$. For this, we begin with a standard discretization argument to bound the operator norm of $(X-\mathbb{E} X)$. [The argument is neither optimal (better constants are possible) nor the quickest (for an expert in probability, appealing to comparison theorems for subgaussian processes [31] would yield the result much faster), but we include it for the sake of completeness.] Let $\mathcal{N}$ be a $\frac{1}{3}$-net in the unit sphere of $\mathbb{C}^{n} \cong \mathbb{R}^{2 n}$ with $|\mathcal{N}| \leq 7^{2 n}$ (see [22, Lemma 2.6] or [32, Lemma 2]), and for $A \in M_{n}^{s a}$ define

$$
\|A\|_{\mathcal{N}}=\sup _{v \in \mathcal{N}}|\langle A v, v\rangle| \text {. }
$$

Then $\|A\|_{\infty} \leq 3\|A\|_{\mathcal{N}}$ by [32, Lemma 4].

For each $u \in S^{n-1}, A \mapsto|\langle A u, u\rangle|$ is a convex and 1-Lipschitz function $M_{n}^{s a} \rightarrow \mathbb{R}$, so by the CCP (11),

$$
\begin{aligned}
\mathbb{P}\left[\|X-\mathbb{E} X\|_{\infty}>t\right] & \leq \mathbb{P}\left[\|X-\mathbb{E} X\|_{\mathcal{N}}>t / 3\right] \\
& \leq \sum_{v \in \mathcal{N}} \mathbb{P}[|\langle(X-\mathbb{E} X) v, v\rangle|>t / 3] \leq C 7^{2 n} e^{-c t^{2}} .
\end{aligned}
$$


From this it follows that $\mathbb{M}\|X-\mathbb{E} X\|_{\infty} \leq C \sqrt{n}$. Since $\|\cdot\|_{\infty} \leq\|\cdot\|_{2}$, the CCP (11) applies to the function $f(A)=\|A\|_{\infty}$, and so $\mathbb{E}\|X-\mathbb{E} X\|_{\infty} \leq C \sqrt{n}$ as well. (Alternatively, this latter estimate follows by combining the inequality above with integration by parts.)

We also have the elementary estimate (a very weak consequence of CCP)

$$
\mathbb{E}\|X-\mathbb{E} X\|_{2} \leq \sqrt{\mathbb{E}\|X-\mathbb{E} X\|_{2}^{2}}=\sqrt{\sum_{i, j} \mathbb{E}\left|x_{i j}-\mathbb{E} x_{i j}\right|^{2}} \leq C n .
$$

From the above estimates and Hölder's inequality, we obtain that for $p \geq 2$,

$$
\begin{aligned}
\mathbb{E}\|X-\mathbb{E} X\|_{p} & \leq \mathbb{E}\left(\|X-\mathbb{E} X\|_{2}^{2 / p}\|X-\mathbb{E} X\|_{\infty}^{1-2 / p}\right) \\
& \leq\left(\mathbb{E}\|X-\mathbb{E} X\|_{2}\right)^{2 / p}\left(\mathbb{E}\|X-\mathbb{E} X\|_{\infty}\right)^{1-2 / p} \\
& \leq C n^{2 / p} n^{1 / 2-1 / p}=C n^{\frac{1}{p}+\frac{1}{2}}
\end{aligned}
$$

Specifying $p=2(d-1)$ yields

$$
\mathbb{E}\|X\|_{2(d-1)} \leq \mathbb{E}\|X-\mathbb{E} X\|_{2(d-1)}+\|\mathbb{E} X\|_{2(d-1)} \leq C n^{d / 2(d-1)} .
$$

(It is here that our hypothesis for noncentered random matrices enters into play and where the form of the hypothesis is clarified.) Now $\|\cdot\|_{2(d-1)} \leq\|\cdot\|_{2}$, so the CCP (11) applies to the function $f(A)=\|A\|_{2(d-1)}$. This implies finally that for $a=n^{d / 2(d-1)} b, b \geq C$,

$$
\mathbb{P}\left[X \notin K_{a}\right]=\mathbb{P}\left[\|X\|_{2(d-1)} \geq a\right] \leq C \exp \left[-c n^{d /(d-1)} b^{2}\right]
$$

We are now ready to carry out the argument to bound the tails of $(F(X)-$ $\mathbb{M} F(X))$ by — in particular — appropriately choosing the truncation level $a$. Recall that $F_{a}: M_{n}^{s a} \rightarrow \mathbb{R}$ are the functions provided by Lemma 5 . The monotonicity in $a$ of $F_{a}(A)$ implies that $\mathbb{M} F_{a}(X)$ increases in $a$ to $\mathbb{M} F(X)$. Letting $a=C_{1} n^{d / 2(d-1)}$ and applying the CCP (11) to $F_{a}$ we obtain

$$
\begin{aligned}
& \mathbb{P}\left[F(X) \geq \mathbb{M} F_{a}(X)+s\right]= \mathbb{P}\left[\left(F_{a}(X) \geq \mathbb{M} F_{a}(X)+s\right) \text { and }\left(X \in K_{a}\right)\right] \\
&+\mathbb{P}\left[\left(F(X) \geq \mathbb{M} F_{a}(X)+s\right) \text { and }\left(X \notin K_{a}\right)\right] \\
& \leq \mathbb{P}\left[F_{a}(X) \geq \mathbb{M} F_{a}(X)+s\right]+\mathbb{P}\left[X \notin K_{a}\right] \\
& \leq C \exp \left[-c \frac{s^{2}}{d^{2} C_{1}^{2(d-1)} n^{d}}\right]+C \exp \left[-c n^{d /(d-1)} C_{1}^{2}\right] .
\end{aligned}
$$

Therefore if $C_{1}$ is chosen large enough (independently of $n$ and $d$ ), then

$$
\mathbb{P}\left[F(X) \geq \mathbb{M} F_{a}(X)+C_{2} d C_{1}^{d-1} n^{d / 2}\right]<\frac{1}{2}
$$

for some $C_{2}>0$, and so $\mathbb{M} F(X) \leq \mathbb{M} F_{a}(X)+d C_{3}^{d} n^{d / 2}$. Since $\mathbb{M} F_{a}(X)$ increases monotonically with $a$, we obtain

$$
\left|\mathbb{M} F(X)-\mathbb{M} F_{a}(X)\right| \leq d C_{3}^{d} n^{d / 2}
$$

for every $a \geq C_{1} n^{d / 2(d-1)}$. (This is the point at which it is most convenient to be working with the median instead of the mean, since for a fixed $a$ the bound we get for $\mathbb{P}\left[\left|F(X)-\mathbb{M} F_{a}(X)\right| \geq s\right]$ is not integrable.) 
Now set $a=b n^{d / 2(d-1)}$ with $b \geq C_{1}$. For $s \geq 2 d C_{3}^{d} n^{d / 2}$, by applying the CCP (11) to $F_{a}$ again,

$$
\begin{aligned}
\mathbb{P}[|F(X)-\mathbb{M} F(X)| \geq s]= & \left.\mathbb{P}\left[\left|F_{a}(X)-\mathbb{M} F(X)\right| \geq s\right) \text { and }\left(X \in K_{a}\right)\right] \\
& \left.+\mathbb{P}[|F(X)-\mathbb{M} F(X)| \geq s) \text { and }\left(X \notin K_{a}\right)\right] \\
\leq & \mathbb{P}\left[\left|F_{a}(X)-\mathbb{M} F_{a}(X)\right| \geq\left(s-d C_{3}^{d} n^{d / 2}\right)\right]+\mathbb{P}\left[X \notin K_{a}\right] \\
\leq & C \exp \left[-c \frac{\left(s-d C_{3}^{d} n^{d / 2}\right)^{2}}{d^{2} a^{2(d-1)}}\right]+C \exp \left[-c n^{d /(d-1)} b^{2}\right] \\
\leq & C \exp \left[-c \frac{s^{2}}{d^{2} b^{2(d-1)} n^{d}}\right]+C \exp \left[-c n^{d /(d-1)} b^{2}\right] .
\end{aligned}
$$

If $s \leq C_{1}^{d} d n^{d^{2} / 2(d-1)}$ and $b=C_{1}$, then the first term in the last estimate dominates the second. If $s \geq C_{1}^{d} d n^{d^{2} / 2(d-1)}$, then setting $b=d^{-1} n^{-d / 2(d-1)} s^{1 / d}$ results in both exponents being of the same order, and we obtain

$$
\mathbb{P}[|F(X)-\mathbb{M} F(X)| \geq s] \leq C \exp \left[-\min \left\{\frac{s^{2}}{d^{2}\left(C^{\prime} n\right)^{d}}, c s^{2 / d}\right\}\right]
$$

for all $s \geq 2 d C_{3}^{d} n^{d / 2}$. The inequality above is vacuously true (with appropriately chosen constants) if $s<2 d C_{3}^{d} n^{d / 2}$. Finally, substituting $s=n^{d / 2} t$ yields the bound in the statement of the proposition.

Parts of the analysis of this section can be performed for functionals more general than traces of powers, e.g., $A \mapsto \operatorname{tr} \phi(A)$ for $\phi: \mathbb{R} \rightarrow \mathbb{R}$ a convex Lipschitz function as already considered in [11. In an even less restrictive framework, by replacing the convexity lemma [3, Lemma 4.4.12] used above and in [11] with the more general result of [6], one can consider functionals of the form $A \mapsto f\left(\lambda_{1}(A), \ldots, \lambda_{n}(A)\right)$ for a symmetric, convex, Lipschitz function $f: \mathbb{R}^{n} \rightarrow \mathbb{R}$; see [19, Corollary 8.23].

\section{The general Case: Polarization and other tricks}

To deduce a version of Proposition 4 for non-Hermitian matrices, we use the following polarization identity.

Lemma 6. For any $A, B \in M_{n}$,

$$
A^{d}=\frac{1}{d+1} \sum_{j=0}^{d}\left(A+e^{2 \pi i j /(d+1)} B\right)^{d} .
$$

In particular,

$$
A^{d}=\frac{1}{d+1} \sum_{j=0}^{d} e^{\pi i j d /(d+1)}\left(e^{-\pi i j /(d+1)} A+e^{\pi i j /(d+1)} A^{*}\right)^{d} .
$$

Proof. Expanding the sum, there are matrices $M_{k}, k=0, \ldots, d$, with $M_{0}=A^{d}$, such that

$$
\left(A+e^{2 \pi i j /(d+1)} B\right)^{d}=\sum_{k=0}^{d} e^{2 \pi i j k /(d+1)} M_{k} .
$$


The $(d+1) \times(d+1)$ Fourier matrix $\left[\frac{1}{\sqrt{d+1}} e^{2 \pi i j k /(d+1)}\right]_{j, k=0}^{d}$ is unitary, so inverting the above relations yields

$$
M_{k}=\frac{1}{d+1} \sum_{j=0}^{d} e^{-2 \pi i j k /(d+1)}\left(A+e^{2 \pi i j /(d+1)} B\right)^{d} .
$$

The lemma is the case $k=0$ of this identity.

Corollary 7. Let $X \in M_{n}$ be a random matrix which satisfies the convex concentration property (with respect to the Hilbert-Schmidt norm on $M_{n}$ ), let $d \geq 1$ be an integer, and suppose - when $d \geq 2-$ that $\|\mathbb{E} X\|_{2(d-1)} \leq c n^{d / 2(d-1)}$. Then for $t>0$,

$$
\mathbb{P}\left[\left|\operatorname{tr}\left(\frac{X}{\sqrt{n}}\right)^{d}-\mathbb{E} \operatorname{tr}\left(\frac{X}{\sqrt{n}}\right)^{d}\right| \geq t\right] \leq C(d+1) \exp \left[-\min \left\{c^{d} t^{2}, c n t^{2 / d}\right\}\right] .
$$

Proof. Observe that for any $\theta \in \mathbb{R}, A \mapsto e^{-i \theta} A+e^{i \theta} A^{*}$ is a 2-Lipschitz map $M_{n} \rightarrow M_{n}^{s a}$. Thus $Y_{\theta}=e^{-i \theta} X+e^{i \theta} X^{*}$ satisfies the hypotheses of Proposition 4. As remarked earlier, in the conclusion of Proposition 4, the median may be replaced by the mean. Set $\theta_{j}=\pi j /(d+1)$ for $j=0,1, \ldots, d$. Then, by Lemma 6 , $\left(\frac{X}{\sqrt{n}}\right)^{d}=\frac{1}{d+1} \sum_{j=0}^{d} e^{i d \theta_{j} /(d+1)}\left(\frac{Y_{\theta_{j}}}{\sqrt{n}}\right)^{d}$, and hence, by Proposition 4 ,

$$
\begin{aligned}
\mathbb{P}\left[\left|\operatorname{tr}\left(\frac{X}{\sqrt{n}}\right)^{d}-\mathbb{E} \operatorname{tr}\left(\frac{X}{\sqrt{n}}\right)^{d}\right| \geq t\right] & \leq \mathbb{P}\left[\frac{1}{d+1} \sum_{j=0}^{d}\left|\operatorname{tr}\left(\frac{Y_{\theta_{j}}}{\sqrt{n}}\right)^{d}-\mathbb{E} \operatorname{tr}\left(\frac{Y_{\theta_{j}}}{\sqrt{n}}\right)^{d}\right| \geq t\right] \\
& \leq(d+1) \sup _{\theta \in \mathbb{R}} \mathbb{P}\left[\left|\operatorname{tr}\left(\frac{Y_{\theta}}{\sqrt{n}}\right)^{d}-\mathbb{E} \operatorname{tr}\left(\frac{Y_{\theta}}{\sqrt{n}}\right)^{d}\right| \geq t\right] \\
& \leq C(d+1) \exp \left[-\min \left\{c^{d} t^{2}, c n t^{2 / d}\right\}\right] .
\end{aligned}
$$

Proof of Theorem 1. By the triangle inequality, it suffices to consider the case when $P$ is a noncommutative $*$-monomial. (Note that for fixed $m$ and $d$ there are, up to scalar multiples, only finitely many distinct noncommutative $*$-monomials of degree at most $d$ in $m$ variables.) Write $P\left(x_{1}, \ldots, x_{m}\right)=y_{1} \ldots y_{d}$, where each $y_{j}$ is equal to some $x_{k}$ or $x_{l}^{*}$, and then define

$$
X=\left[\begin{array}{ccccc}
0 & Y_{1} & & & \\
& 0 & Y_{2} & & \\
& & \ddots & \ddots & \\
& & & 0 & Y_{d-1} \\
Y_{d} & & & & 0
\end{array}\right]
$$

analogously. It is easy to verify that

$$
X^{d}=\left[\begin{array}{cccc}
Y_{1} Y_{2} \cdots Y_{d} & & & 0 \\
& Y_{2} Y_{3} \cdots Y_{d} Y_{1} & & \\
& & \ddots & \\
0 & & & Y_{d} Y_{1} \cdots Y_{d-1}
\end{array}\right]
$$

so that $\operatorname{tr} X^{d}=d \operatorname{tr} P\left(X_{1}, \ldots, X_{m}\right)$. Furthermore, $X$ satisfies the convex concentration property on $M_{d n}$, with constants that may now depend on $d$ (cf. [19, Proposition 1.11]). The theorem now follows by applying Corollary 7 to $X$. 


\section{ACKNOWLEDGEMENTS}

This research has been partially supported by the authors' respective grants from the National Science Foundation (USA). Early versions of the results have been disseminated in various venues since 2005. The authors thank W. Bryc and G. Kuperberg for inspiring conversations. The second-named author thanks Institut Mittag-Leffler, where he was in residence while the final version of this paper was being written.

\section{REFERENCES}

1. R. Adamczak, Logarithmic Sobolev inequalities and concentration of measure for convex functions and polynomial chaoses, Bull. Pol. Acad. Sci. Math. 53 (2005), no. 2, 221-238. MR 2163396 (2006h:60028)

2. N. Alon, M. Krivelevich, and V. H. Vu, On the concentration of eigenvalues of random symmetric matrices, Israel J. Math. 131 (2002), 259-267. MR.1942311(2003i:15020)

3. G. W. Anderson, A. Guionnet, and O. Zeitouni, An introduction to random matrices, Cambridge Studies in Advanced Mathematics, vol. 118, Cambridge University Press, 2009. MR.2760897

4. M. Capitaine and C. Donati-Martin, Strong asymptotic freeness for Wigner and Wishart matrices, Indiana Univ. Math. J. 56 (2007), no. 2, 767-803. MR2317545 (2008e:46079)

5. K. R. Davidson and S. J. Szarek, Local operator theory, random matrices and Banach spaces, Handbook of the Geometry of Banach Spaces, Vol. I, North-Holland, Amsterdam, 2001, pp. 317-366. MR.1863696 (2004f:47002a)

6. C. Davis, All convex invariant functions of hermitian matrices, Arch. Math. 8 (1957), 276278. MR0090572(19:832b)

7. V. H. de la Peña and S. J. Montgomery-Smith, Bounds on the tail probability of U-statistics and quadratic forms, Bull. Amer. Math. Soc. (N.S.) 31 (1994), no. 2, 223-227. MR1261237 $(95 \mathrm{j}: 60032)$

8. P. Deift and D. Gioev, Random matrix theory: Invariant ensembles and universality, Courant Lecture Notes in Mathematics, vol. 18, Courant Institute of Mathematical Sciences, New York, 2009. MR2514781

9. K. Dykema, On certain free product factors via an extended matrix model, J. Funct. Anal. 112 (1993), no. 1, 31-60. MR.1207936 (95a:46085)

10. A. Guionnet, Large random matrices: Lectures on macroscopic asymptotics, Lecture Notes in Mathematics, vol. 1957, Springer-Verlag, Berlin, 2009. MR2498298 (2010d:60018)

11. A. Guionnet and O. Zeitouni, Concentration of the spectral measure for large matrices, Electron. Comm. Probab. 5 (2000), 119-136 (electronic). MR1781846 (2001k:15035)

12. U. Haagerup and S. Thorbjørnsen, A new application of random matrices: $\operatorname{Ext}\left(C_{\mathrm{red}}^{*}\left(F_{2}\right)\right)$ is not a group, Ann. of Math. (2) 162 (2005), no. 2, 711-775. MR2183281 (2009k:46121)

13. F. Hiai and D. Petz, Asymptotic freeness almost everywhere for random matrices, Acta Sci. Math. (Szeged) 66 (2000), no. 3-4, 809-834. MR1804226 (2002c:15042)

14. The semicircle law, free random variables and entropy, Mathematical Surveys and Monographs, vol. 77, American Mathematical Society, Providence, RI, 2000. MR1746976 (2001j:46099)

15. S. Janson, Poisson approximation for large deviations, Random Structures Algorithms 1 (1990), no. 2, 221-229. MR1138428 (93a:60041)

16. J. H. Kim and V. H. Vu, Concentration of multivariate polynomials and its applications, Combinatorica 20 (2000), no. 3, 417-434. MR.1774845 (2002b:05123)

17. M. Krivelevich and V. H. Vu, Approximating the independence number and the chromatic number in expected polynomial time, J. Comb. Optim. 6 (2002), no. 2, 143-155. MR 1885488 (2002m:05181)

18. R. Latała, Estimates of moments and tails of Gaussian chaoses, Ann. Probab. 34 (2006), no. 6, 2315-2331. MR.2294983 (2008a:60045)

19. M. Ledoux, The concentration of measure phenomenon, Mathematical Surveys and Monographs, vol. 89, American Mathematical Society, Providence, RI, 2001. MR.1849347 (2003k:28019) 
20. C. Male, Norm of polynomials in large random and deterministic matrices, preprint, available at http://arxiv.org/abs/1004.4155, 2010.

21. M. W. Meckes, Concentration of norms and eigenvalues of random matrices, J. Funct. Anal. 211 (2004), no. 2, 508-524. MR2057479 (2005c:46109)

22. V. D. Milman and G. Schechtman, Asymptotic theory of finite-dimensional normed spaces, Lecture Notes in Mathematics, vol. 1200, Springer-Verlag, Berlin, 1986. MR856576 (87m:46038)

23. J. A. Mingo, P. Śniady, and R. Speicher, Second order freeness and fluctuations of random matrices. II. Unitary random matrices, Adv. Math. 209 (2007), no. 1, 212-240. MR2294222 (2009c:15027)

24. J. A. Mingo and R. Speicher, Second order freeness and fluctuations of random matrices. I. Gaussian and Wishart matrices and cyclic Fock spaces, J. Funct. Anal. 235 (2006), no. 1, 226-270. MR2216446 (2007h:46080)

25. T. Oraby, The spectral laws of Hermitian block-matrices with large random blocks, Electron. Comm. Probab. 12 (2007), 465-476. MR2365648 (2008m:60038)

26. R. T. Rockafellar, Convex analysis, Princeton Mathematical Series, No. 28, Princeton University Press, Princeton, NJ, 1970. MR0274683 (43:445)

27. H. Schultz, Non-commutative polynomials of independent Gaussian random matrices. The real and symplectic cases, Probab. Theory Related Fields 131 (2005), no. 2, 261-309. MR2117954 (2005k:46173)

28. M. Talagrand, An isoperimetric theorem on the cube and the Kintchine-Kahane inequalities, Proc. Amer. Math. Soc. 104 (1988), no. 3, 905-909. MR964871 (90h:60016)

29. _ Concentration of measure and isoperimetric inequalities in product spaces, Inst. Hautes Études Sci. Publ. Math. 81 (1995), 73-205. MR1361756 (97h:60016)

30. New concentration inequalities in product spaces, Invent. Math. 126 (1996), no. 3, 505-563. MR1419006 (99b:60030)

31. The generic chaining, Springer Monographs in Mathematics, Springer-Verlag, Berlin, 2005. MR2133757 (2006b:60006)

32. R. Vershynin, Introduction to the non-asymptotic analysis of random matrices, Compressed Sensing: Theory and Applications (Y. Eldar and G. Kutyniok, eds.), Cambridge University Press, to appear. Preprint available at http://arxiv.org/abs/1011.3027.

33. D. V. Voiculescu, Limit laws for random matrices and free products, Invent. Math. 104 (1991), no. 1, 201-220. MR.1094052 (92d:46163)

34. D. V. Voiculescu, K. J. Dykema, and A. Nica, Free random variables, CRM Monograph Series, vol. 1, American Mathematical Society, Providence, RI, 1992. MR1217253 (94c:46133)

35. V. H. Vu, On the concentration of multivariate polynomials with small expectation, Random Structures Algorithms 16 (2000), no. 4, 344-363. MR.1761580 (2001g:60060)

Department of Mathematics, Case Western Reserve University, Cleveland, Ohio 44106-7058

E-mail address: mark.meckes@case.edu

Department of Mathematics, Case Western Reserve University, Cleveland, Ohio 44106-7058 - and - Université Pierre et Marie Curie, Institut de Mathématiques de Jussieu (Equipe d’Analyse Fonctionnelle), BC 247, 4 Place Jussieu, 75252 Paris Cedex 05, France

E-mail address: szarek@cwru.edu 